\title{
Technical feasibility of constant-load and high-intensity interval training for cardiopulmonary conditioning using a re-engineered dynamic leg press
}

\author{
Farouk Chrif ${ }^{1,2^{*}}$, Tobias $\mathrm{Nef}^{2}$ and Kenneth $\mathrm{J} \mathrm{Hunt}^{1}$
}

\begin{abstract}
Background: Leg-press devices are one of the most widely used training tools for musculoskeletal strengthening of the lower-limbs, and have demonstrated important cardiopulmonary benefits for healthy and patient populations. Further engineering development was done on a dynamic leg-press for work-rate estimation by integrating force and motion sensors, power calculation and a visual feedback system for volitional work-rate control. This study aimed to assess the feasibility of the enhanced dynamic leg press for cardiopulmonary exercise training in constant-load training and high-intensity interval training. Five healthy participants aged $31.0 \pm 3.9$ years (mean \pm standard deviation) performed two cardiopulmonary training sessions: constant-load training and high-intensity interval training. Participants carried out the training sessions at a work rate that corresponds to their first ventilatory threshold for constant-load training, and their second ventilatory threshold for high-intensity interval training.

Results: All participants tolerated both training protocols, and could complete the training sessions with no complications. Substantial cardiopulmonary responses were observed. The difference between mean oxygen uptake and target oxygen uptake was $0.07 \pm 0.34 \mathrm{~L} / \mathrm{min}(103 \pm 17 \%)$ during constant-load training, and $0.35 \pm 0.66 \mathrm{~L} / \mathrm{min}$ $(113 \pm 27 \%)$ during high-intensity interval training. The difference between mean heart rate and target heart rate was $-7 \pm 19$ bpm (94 $\pm 15 \%)$ during constant-load training, and $4.2 \pm 16$ bpm (103 $\pm 12 \%)$ during high-intensity interval training.
\end{abstract}

Conclusions: The enhanced dynamic leg press was found to be feasible for cardiopulmonary exercise training, and for exercise prescription for different training programmes based on the ventilatory thresholds.

Keywords: Cardiorespiratory fitness, Resistance training, Exercise test, Exercise, High-intensity interval training, Heart rate, Anaerobic threshold, Feedback, Sensory

\section{Background}

It has been shown that exercise training has numerous health benefits both for healthy individuals and for patient populations, and physical inactivity can increases the risks of chronic diseases [1-3]. These health benefits are

\footnotetext{
*Correspondence: farouk.chrif@bfh.ch

${ }^{1}$ Institute for Rehabilitation and Performance Technology, Division of Mechanical Engineering, Department of Engineering and Information Technology, Bern University of Applied Sciences, CH-3400 Burgdorf, Switzerland

${ }^{2}$ Gerontechnology and Rehabilitation Research Group, ARTORG Center for Biomedical Engineering Research, University of Bern, $\mathrm{CH}-3008$ Bern, Switzerland
}

often associated with higher cardiorespiratory fitness levels (peak oxygen uptake $\dot{V} \mathrm{O}_{2 \text { peak }}$, peak heart rate $\mathrm{HR}_{\text {peak }}$ ), which can be improved and increased by regular exercise training. A complete training programme should include aerobic exercise, muscular fitness, and flexibility exercise, and it is important to be well designed following exercise prescription guidelines (frequency, intensity, and duration) to meet an individual's health and physical fitness goals. The recommended exercise prescription for most adults is an accumulation of at least 150 min.wk ${ }^{-1}$ at moderate intensity, or $75 \mathrm{~min}^{-\mathrm{wk}^{-1}}$ at vigorous intensity [4].

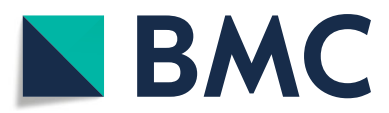

(c) The Author(s). 2019 Open Access This article is distributed under the terms of the Creative Commons Attribution 4.0 International License (http://creativecommons.org/licenses/by/4.0/), which permits unrestricted use, distribution, and reproduction in any medium, provided you give appropriate credit to the original author(s) and the source, provide a link to the Creative Commons license, and indicate if changes were made. The Creative Commons Public Domain Dedication waiver (http://creativecommons.org/publicdomain/zero/1.0/) applies to the data made available in this article, unless otherwise stated. 
The most widely adopted exercise training techniques in clinical and sports environments are constant-load training (CLT) and high-intensity interval training (HIIT). A CLT programme consists of a continuous training phase at light (57-63\% of $\mathrm{HR}_{\text {peak }}$, or $37-45 \%$ of $\dot{V} \mathrm{O}_{2 \text { peak }}$ ) to moderate intensity (64-76\% of $\mathrm{HR}_{\text {peak }}$, or $46-63 \%$ of $\dot{V \mathrm{O}_{2 p e a k}}$ ) for at least $30 \mathrm{~min}$ [4]. CLT is often recommended for patient populations, because of high safety and tolerability [5]. On the other hand, HIIT can be defined as repeated short bouts of vigorous intensity training (77-95\% of $\mathrm{HR}_{\text {peak }}$, or 64-90\% of $\dot{V} \mathrm{O}_{2 \text { peak }}$ ). The duration of the intervals can vary from $5 \mathrm{~s}$ to $8 \mathrm{~min}$, depending on the nature of the exercise and the training device used [4, 6]. HIIT has been proven to produce improvements in physiological power for both healthy $[7,8]$ and patient $[9,10]$ populations, and it is often considered as a time-efficient training strategy, where some studies reported that programmes as short as two weeks' duration could elicit substantial increases in $\dot{V} \mathrm{O}_{\text {2peak }}[8,11]$.

The prescription of a correct exercise intensity is important and has to be tailored individually depending on the person's capacity. It is either related to a percentage of peak capacity $\left(\dot{V} \mathrm{O}_{2 \text { peak }}, \mathrm{HR}_{\text {peak }}\right)$ or linked to the ventilatory thresholds (first ventilatory threshold VT1, and second ventilatory threshold VT2) [12]. The use of ventilatory thresholds for exercise prescription is recommended [13-15] because, unlike the maximal capacity percentage, ventilatory thresholds are independent of participants' motivation [16] and the duration of the exercise testing protocol [17]. It was reported previously that a dynamic leg-press is feasible for incremental cardiopulmonary exercise testing, and the substantial cardiopulmonary responses allowed the identification of peak and sub-maximal parameters with a high rate of success [18].

Leg-press training devices allow the performance of resistive exercise, where eccentric and concentric work regimes are combined $[18,19]$; this has been proven to provide important physiological benefits [20-22]. They are mainly used for musculoskeletal conditioning [23], and showed potential for neuromuscular rehabilitation applications [24]. A previous study evaluated changes in $\dot{V} \mathrm{O}_{2 \text { peak }}$ and found that a leg press exercise training programme elicits larger increases in $\dot{V} \mathrm{O}_{2 \text { peak }}$ when tested on the leg press than when tested on a cycle or a treadmill [25]. Another study reported that leg press exercise elicits cardiovascular benefits when used as resistance training in cardiac patients [26].

To facilitate and to promote active participation during training sessions, further engineering development was necessary on a dynamic leg-press for work-rate estimation by integrating force sensors behind the footplates, motion sensors in the rotation axis of the pedals, power calculation and a visual feedback system. These engineering modifications facilitated accurate work rate calculation, and allowed participants to see their exercise work rate and adjust their volitional effort to meet the target work rate. This approach was previously shown to be feasible for incremental cardiopulmonary exercise testing in healthy participants [18].

The aim of this study was to assess the feasibility of the re-engineered dynamic leg press for cardiopulmonary exercise training (CLT and HIIT programmes) in healthy males. Feasibility assessment considered: (i) technical implementation, (ii) acceptability and (iii) responsiveness.

\section{Results}

Individual outcome data from the 5 participants in the case series are presented in Table 1 and illustrated in Figs. 3, 4, 5, 6, and 7. The data show that in all cases highly accurate work rate control was achieved for all CLT and HIIT target profiles (Figure parts (g) and (h)).

During the CLT, the overall difference, averaged across all participants, between mean steady-state oxygen uptake $\dot{V} \mathrm{O}_{2}$ and $\dot{V} \mathrm{O}_{2 \mathrm{VT} 1}$ was $\Delta \dot{V} \mathrm{O}_{2 \mathrm{VT} 1}=0.07 \pm 0.34 \mathrm{~L} / \mathrm{min}(103$ $\pm 17 \%$, relative to target value) (mean \pm SD). The overall difference between mean steady-state heart rate HR and $\mathrm{HR}_{\mathrm{VT} 1}$ was $\Delta \mathrm{HR}_{\mathrm{VT} 1}=-7 \pm 19 \mathrm{bpm}(94 \pm 15 \%)$.

During the HIIT, the overall difference between the mean of the highest oxygen uptake values during each high-intensity phase $\dot{V} \mathrm{O}_{2}$ and $\dot{V} \mathrm{O}_{2 \mathrm{VT} 2}$ was $\Delta \dot{V} \mathrm{O}_{2 \mathrm{VT} 1}=$ $0.35 \pm 0.66 \mathrm{~L} / \mathrm{min}(113 \pm 27 \%)$. The overall difference between the mean of the highest heart rate values HR and $\mathrm{HR}_{\mathrm{VT} 2}$ was $\Delta \mathrm{HR}_{\mathrm{VT} 2}=4 \pm 16 \mathrm{bpm}(103 \pm 12 \%)$.

The DLP is deemed to be feasible for performing cardiopulmonary exercise training, since the formal feasibility assessment criteria were evaluated as follows:

(i) Implementation: The augmentation of the DLP with work rate estimation and visual feedback enabled the successful implementation of the training programmes (viz. CLT and HIIT).

(ii) Acceptability: All participants tolerated both training protocols, and could complete the training sessions with no complications.

(iii) Responsiveness: Substantial cardiopulmonary responses were observed for both CLT and HIIT: for CLT, the mean steady-state oxygen uptake $\dot{V} \mathrm{O}_{2}$ was $48 \%$ of $\dot{V} \mathrm{O}_{2 \text { peak }}$ and mean steady-state heart rate $\mathrm{HR}$ was $70 \%$ of $\mathrm{HR}_{\text {peak }}$; for HIIT, the mean of the highest oxygen uptake values was $90 \%$ of $\dot{V} \mathrm{O}_{2 \text { peak }}$ and the mean of the highest heart rate values was $91 \%$ of $\mathrm{HR}_{\text {peak. }}$

\section{Discussion}

The aim of this study was to assess the feasibility of a dynamic leg press for cardiopulmonary exercise training (CLT and HIIT programmes) in healthy males. Feasibility assessment considered: (i) technical implementation, (ii) acceptability and (iii) responsiveness. 
Table 1 Cardiopulmonary outcomes

\begin{tabular}{lllllll}
\hline Participant & 1 & 2 & 3 & 4 & 5 & mean \pm SD \\
\hline $\mathrm{CLT}$ & & & & & & \\
$\dot{\mathrm{V}} \mathrm{O}_{2}$ & 1.54 & 1.18 & 1.32 & 1.36 & 2.88 & $1.66 \pm 0.70$ \\
\% of $\dot{V} \mathrm{O}_{2 \text { peak }}$ & 52 & 42 & 40 & 39 & 68 & $48 \pm 12$ \\
$\mathrm{HR}$ & 114 & 121 & 137 & 91 & 156 & $124 \pm 24$ \\
\% of $\mathrm{HR}_{\text {peak }}$ & 76 & 63 & 72 & 56 & 83 & $70 \pm 11$ \\
$\mathrm{HIIT}$ & & & & & & \\
$\dot{\mathrm{V}} \mathrm{O}_{2}$ & 3.67 & 2.51 & 2.62 & 1.93 & 4.21 & $2.99 \pm 0.93$ \\
$\%$ of $\dot{V} \mathrm{O}_{2 \text { peak }}$ & 124 & 90 & 79 & 55 & 100 & $90 \pm 26$ \\
$\mathrm{HR}$ & 164 & 173 & 177 & 118 & 177 & $162 \pm 25$ \\
\% of $\mathrm{HR}_{\text {peak }}$ & 109 & 90 & 92 & 72 & 94 & $91 \pm 13$ \\
\hline
\end{tabular}

For the $\mathrm{CLT}, \dot{\mathrm{V}} \mathrm{O}_{2}$ and $\mathrm{HR}$ are the average oxygen uptake and heart rate obtained during the active phases

For the $\mathrm{HIIT}, \mathrm{V}_{2}$ and $\mathrm{HR}$ are the average of the five peak values obtained from each interval

The DLP is deemed feasible for performing cardiopulmonary exercise training. Regarding the implementation, the augmentation of the DLP with work rate estimation and visual feedback enabled the implementation of the exercise training programmes (viz. CLT and HIIT). In term of acceptability and responsiveness, all participants could understand the task and adapt their volitional leg effort to keep to the target work rate. All participants could complete the training sessions with no complications, and substantial cardiopulmonary responses were observed for both CLT and HIIT.

Four participants performed the training sessions for both CLT and HIIT based on their target $\dot{V} \mathrm{O}_{2 \mathrm{VT} 1}$ and $\dot{V} \mathrm{O}_{2 \mathrm{VT} 2}$ obtained from the previous incremental tests
[18]. One participant (participant 1) could not complete the training sessions based on the target outcomes from the incremental test [18]. It was suspected that these outcomes were overestimated, therefore this participant had to re-perform the incremental test where the outcomes were found to be lower than those obtained from the first incremental test.

For two participants (participants 1 and 5), the values obtained were higher than the recommended range of HIIT (vigorous intensity), and corresponded to "near maximal to maximal" intensity (Table 1). As for the CLT, only participant 5 had outcomes higher than the recommended "light to moderate intensity" (Table 1).

Considering $\dot{V} \mathrm{O}_{2}$ in the HIIT protocol, it was observed that participants 1 and 5 performed at intensities which correspond to $124 \%$ and $100 \%$ of $\dot{V} \mathrm{O}_{2 \text { peak }}$ respectively, while, surprisingly, participant 4 performed at only 55\% of his $\dot{V} \mathrm{O}_{2 \text { peak }}$, which is described as "moderate intensity". The high $\dot{V} \mathrm{O}_{2}$ obtained from participants 1 and 5 may be due to the $\dot{V} \mathrm{O}_{2 \text { peak }}$ obtained from the incremental exercise test being due to muscle fatigue after $\sim 10 \mathrm{~min}$ of exercise [18] and not aerobic exhaustion. During the HIIT bouts, the participants tried to reach the target intensity as quickly as possible and kept exercising for a relatively short duration (2 min) at their VT2 which is near to maximal capacity [12], thus high $\dot{V} \mathrm{O}_{2}$ values were elicited. On the other hand, participant 4, who had low cardiovascular outcomes during CLT and HIIT, seemed to be unfamiliar with this kind of exercise (resistive leg exercise). In general, the fact that the participant is not familiar with the type of exercise impairs the result during the exercise test, and the calculated work load is often too low [27].

Table 2 Participants' characteristics and target data

\begin{tabular}{|c|c|c|c|c|c|c|}
\hline Participant & 1 & 2 & 3 & 4 & 5 & mean $\pm S D$ \\
\hline \multicolumn{7}{|l|}{ Characteristics } \\
\hline Age range (yr) & $30-35$ & $30-35$ & $25-30$ & $25-30$ & $30-35$ & $31.0 \pm 3.9$ \\
\hline Body mass (kg) & 86 & 70 & 81 & 80 & 73 & $78.0 \pm 6.4$ \\
\hline Height (m) & 1.88 & 1.72 & 1.81 & 1.83 & 1.82 & $1.81 \pm 0.06$ \\
\hline BMI $\left(\mathrm{kg} / \mathrm{m}^{2}\right)$ & 24.3 & 23.7 & 24.7 & 23.9 & 22.0 & $23.7 \pm 1.0$ \\
\hline \multicolumn{7}{|c|}{ Incremental test outcomes } \\
\hline \multicolumn{7}{|c|}{ oxygen uptake (L/min) } \\
\hline$\dot{\mathrm{V}} \mathrm{O}_{2 \text { peak }}$ & 2.95 & 2.79 & 3.32 & 3.49 & 4.22 & $3.34 \pm 0.56$ \\
\hline$\dot{\mathrm{V}} \mathrm{O}_{2 \mathrm{VT} 1}$ & 1.57 & 1.05 & 1.51 & 1.56 & 2.26 & $1.59 \pm 0.43$ \\
\hline$\dot{\mathrm{V}} \mathrm{O}_{2 \mathrm{VT} 2}$ & 2.35 & 2.32 & 2.52 & 2.38 & 3.64 & $2.64 \pm 0.56$ \\
\hline \multicolumn{7}{|l|}{ heart rate (bpm) } \\
\hline$H R_{\text {peak }}$ & 150 & 192 & 193 & 163 & 188 & $177 \pm 20$ \\
\hline HRVT1 & 115 & 138 & 144 & 122 & 135 & $131 \pm 12$ \\
\hline $\mathrm{HR}_{\mathrm{VT} 2}$ & 136 & 176 & 172 & 133 & 171 & $158 \pm 21$ \\
\hline
\end{tabular}




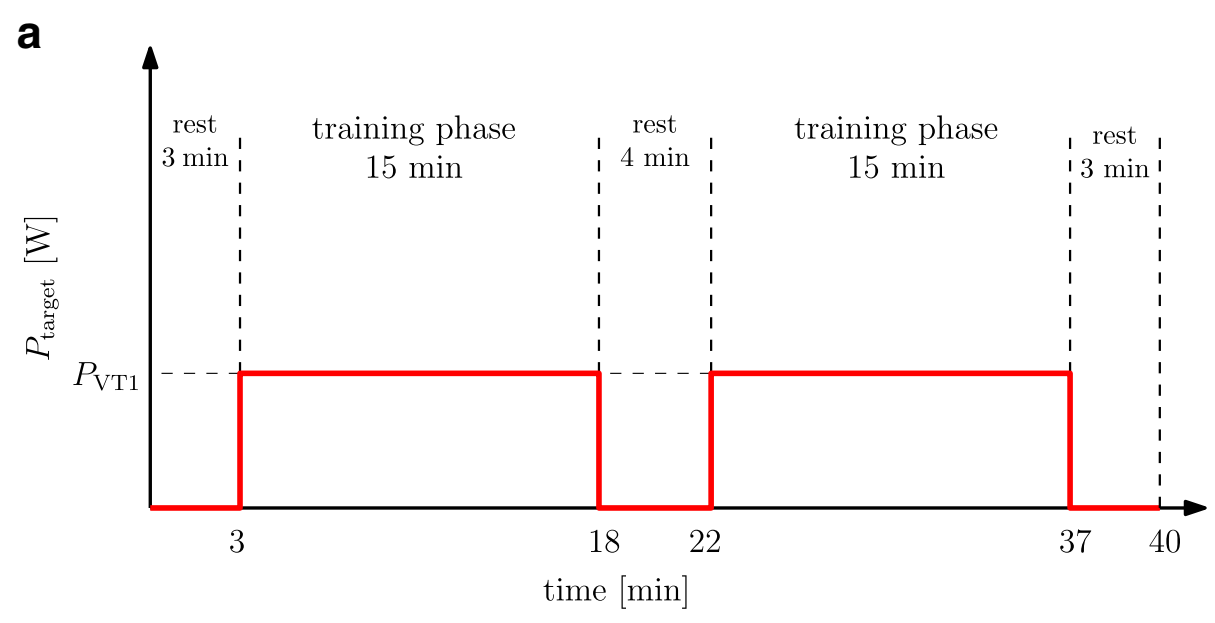

b

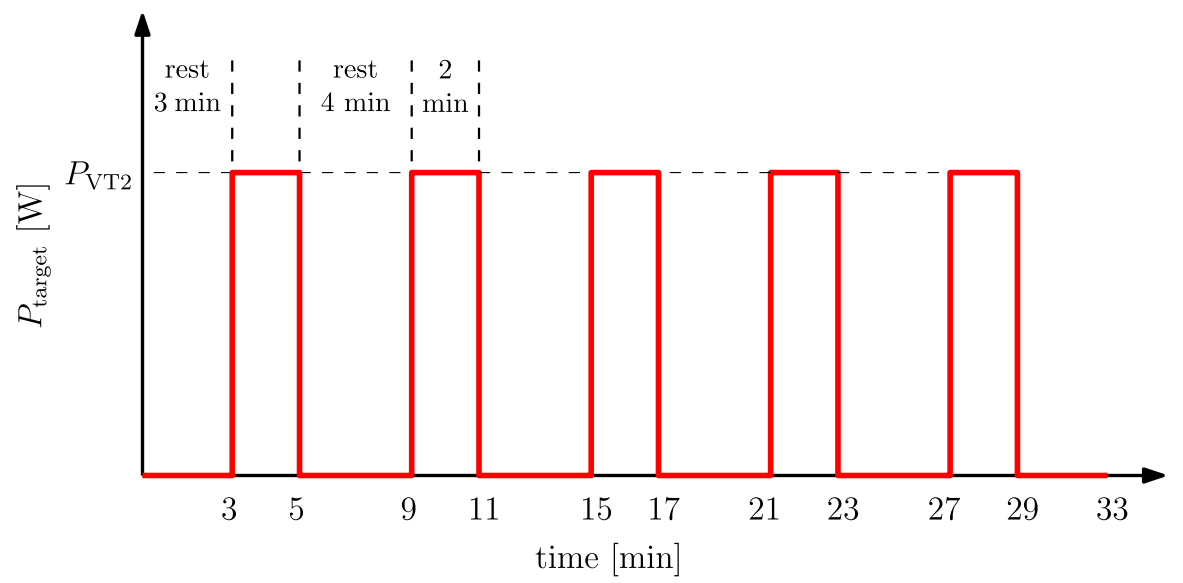

Fig. 1 Exercise testing protocols. $P_{\text {target: }}$ target work rate. a CLT protocol; b HIIT protocol

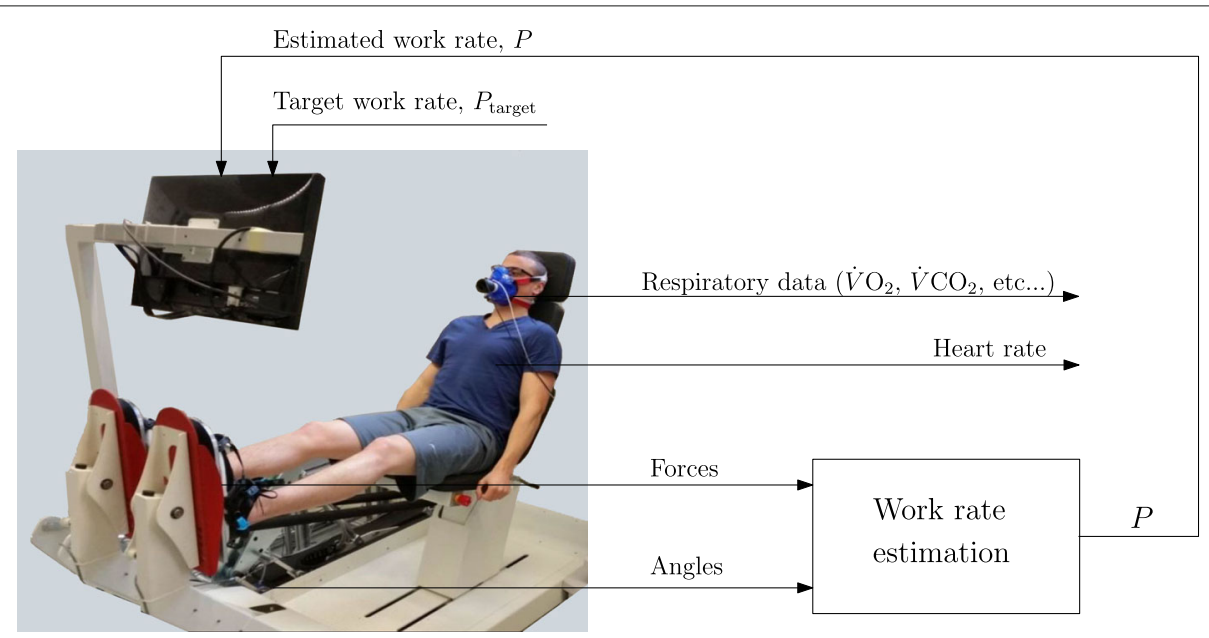

Fig. 2 Dynamic leg press augmented with work rate estimation and visual feedback. (adapted from [18]) 
The test-retest reliability and repeatability of incremental testing using the DLP has not been evaluated, and the predicted peak work rate during the incremental test was adapted from a method developed for a cycle ergometer [28]. As a complement to the present assessment of the DLP for exercise training, future work should include the evaluation of test-retest reliability of cardiopulmonary exercise testing using the DLP, the establishment of a method to predict peak work rate during incremental tests, and the investigation of physiological adaptations to long term training programmes for healthy and patient populations.

\section{Conclusions}

The dynamic leg press was found to be feasible for cardiopulmonary exercise training, and for exercise prescription for different training programmes (viz. CLT and HIIT) based on the ventilatory thresholds.

\section{Methods}

Research participants and study design

This feasibility study was reviewed and approved by the Ethics Review Board of the Canton of Bern in Switzerland (Kantonale Ethikkommission Bern, KEK. Ref. No. 201601502). Written informed consent was obtained from all participants.

\section{CLT}

(a) oxygen uptake

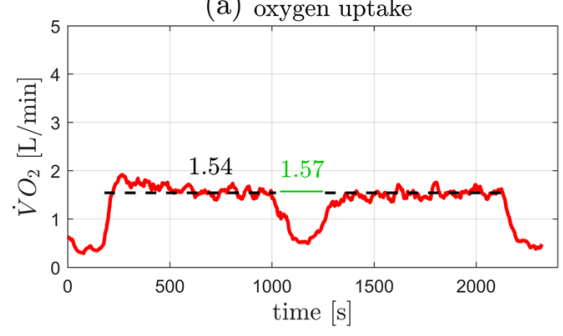

(c) heart rate

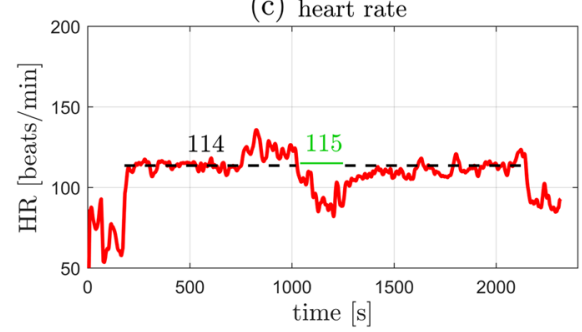

(e) RER

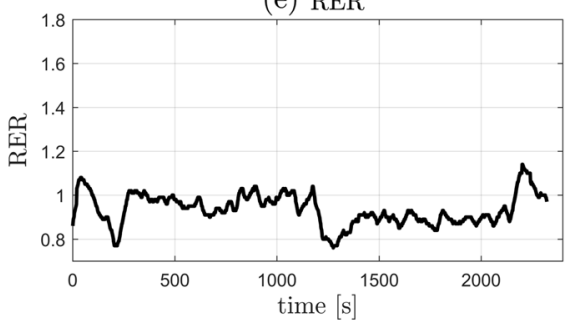

(g) work rate

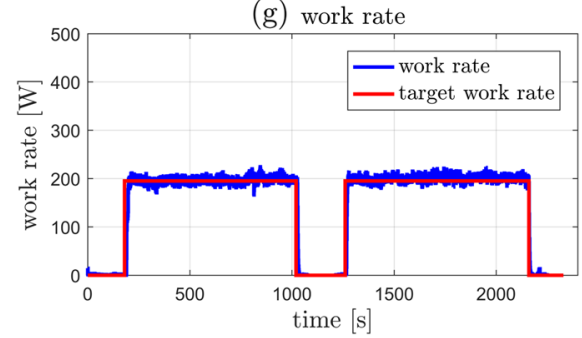

HIIT

(b) oxygen uptake

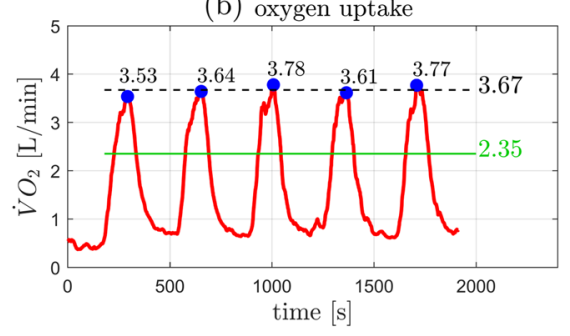

(d) heart rate

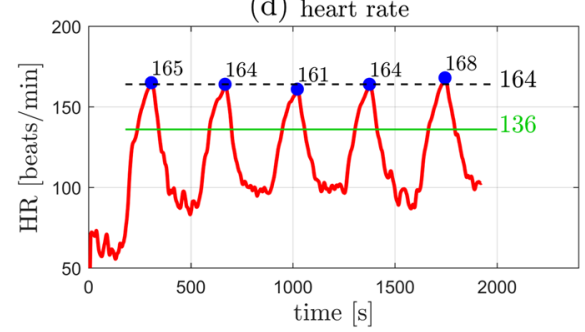

(f) RER

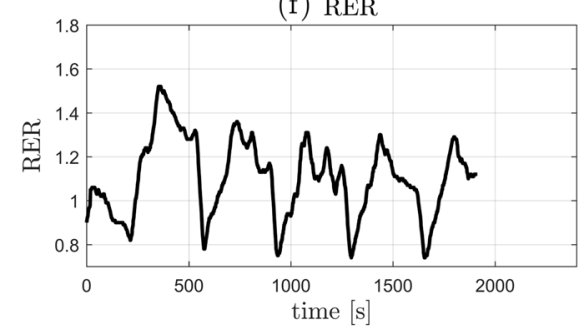

(h) work rate

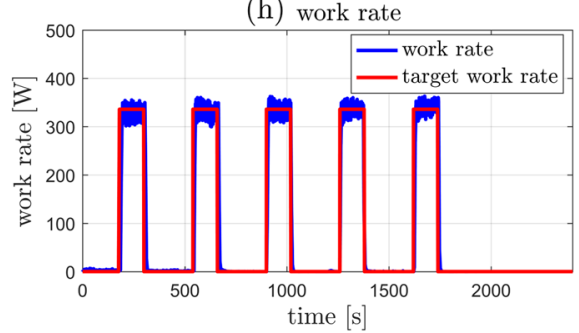

Fig. 3 Original data records from participant 1. Left: CLT, Right: HIIT. (a,b) Oxygen uptake $\dot{V} \mathrm{O}_{2}$. (c,d) Heart rate HR. (e,f) Respiratory exchange ratio (RER). (g,h) Target and measured work rates $\left(P_{\text {target }}, P\right)$. Parts $(\mathbf{a})-(\mathbf{d})$ : the green line indicates the target values, $\dot{V} \mathrm{O}_{2 V T 1}$ for the $\mathrm{CLT}_{\text {and }} \dot{V} \mathrm{O}_{2 V T 2}$ for the HIIT (Table 2); the dashed black line indicates the measured mean value, the steady state mean value for CLT, and the average of the five peak values for HIIT (Table 1). Parts (b),(d): the blue dots indicate the peak values of each interval during HIIT 
Five healthy males (age $31.0 \pm 3.9$ ), all non-smokers with no known cardiovascular, pulmonary or musculoskeletal problems participated (Table 2). The five participants had been recruited among participants in a previous study, where they performed incremental cardiopulmonary exercise tests on the same training device [18]. The two studies were separated by 7 months. Peak cardiopulmonary outcomes ( $\dot{V} \mathrm{O}_{2 \text { peak }}$ and $\left.\mathrm{HR}_{\text {peak }}\right)$, and sub-maximal cardiopulmonary thresholds (first and second ventilatory thresholds $\dot{V} \mathrm{O}_{2 \mathrm{VT} 1}, \dot{V} \mathrm{O}_{2 \mathrm{VT} 2}$, and heart rates at the first and second ventilatory thresholds $\mathrm{HR}_{\mathrm{VT} 1}$, $\mathrm{HR}_{\mathrm{VT} 2}$ ) obtained from the previous study were used for the individual training prescription and analysis (Table 2).
Each participant carried out 2 formal training sessions: (i) a constant-load test (CLT), where participants trained for $30 \mathrm{~min}$ at their personal $P_{\mathrm{VT} 1}$ (work rate that corresponds to $\dot{V} \mathrm{O}_{2 \mathrm{VT}}$ obtained from the incremental test); (ii) a high-intensity interval training (HIIT) session, where participants performed 5 high intensity intervals at their personal $P_{\mathrm{VT} 2}$ (work rate that corresponds to $\dot{V} \mathrm{O}_{2 V T 2}$ obtained from the incremental test). Training sessions were separated by at least $48 \mathrm{~h}$. Participants were required to avoid strenuous activity within the $24 \mathrm{~h}$ prior to each formal test session, to refrain from caffeine for 12 $\mathrm{h}$ before, and not to consume a large meal within $3 \mathrm{~h}$ prior to testing.

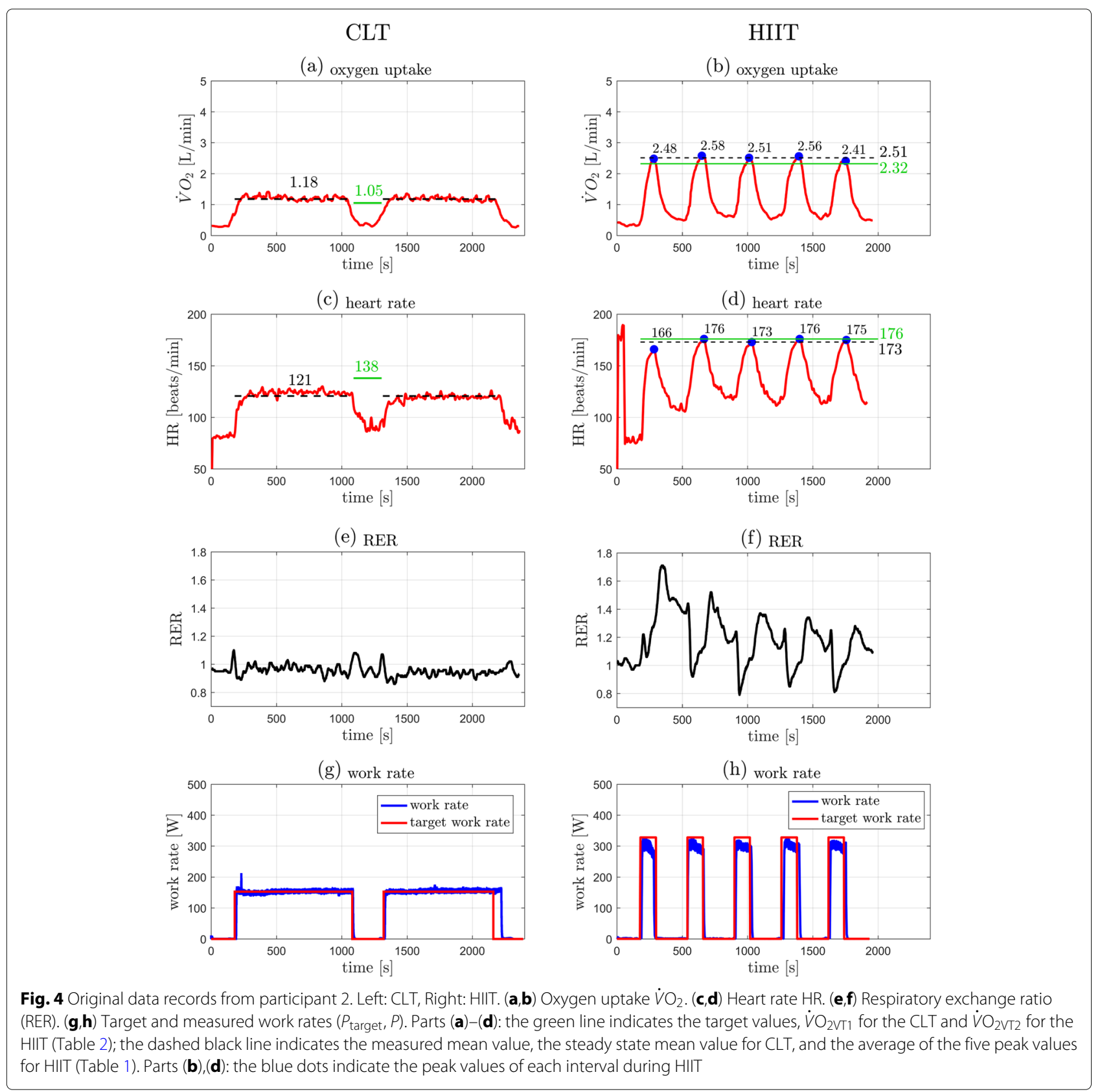


The CLT protocol (Fig. 1a) consisted of 5 stages: a 3-min recorded rest; 2 training phases of 15 min each separated by a 4-min rest period; and a 3-min recorded rest. During the training phases participants were instructed to follow a predefined target work rate $P_{\mathrm{VT} 1}$ which corresponds to their individual $\dot{V} \mathrm{O}_{2 \mathrm{VT} 1}$.

The HIIT protocol (Fig. 1b) started with a 3-min recorded rest, then 5 training intervals of $2 \mathrm{~min}$ at a work rate $P_{\mathrm{VT} 2}$ corresponding to the individual $\dot{V} \mathrm{O}_{2 \mathrm{VT} 2}$, interspersed by 4-min rest periods. The HIIT session was ended by a 3-min recorded rest. For both CLT and HIIT, participants performed 2 min of unrecorded warm-up at low intensity prior to the training session.

\section{Equipment}

A commercial dynamic leg press exercise device (Allegro, Dynamic Devices AG, Switzerland) was used in this study. The dynamic leg press is pneumatically-actuated and was augmented with force sensors in the foot plates, angle sensors at the rotation axis of the pedal, a work rate estimation algorithm, and a visual feedback (Fig. 2).

\section{CLT}

(a) oxygen uptake

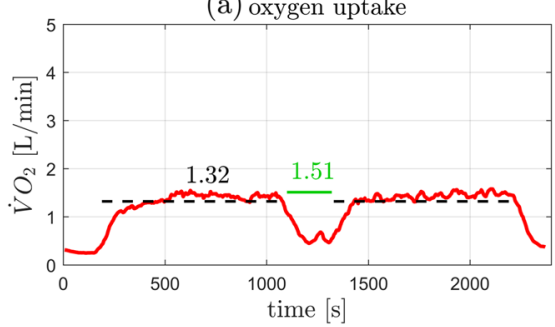

(c) heart rate

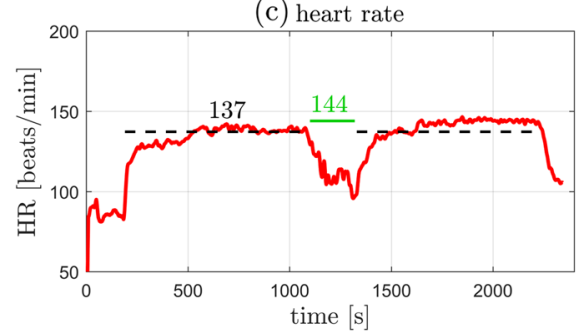

(e) RER

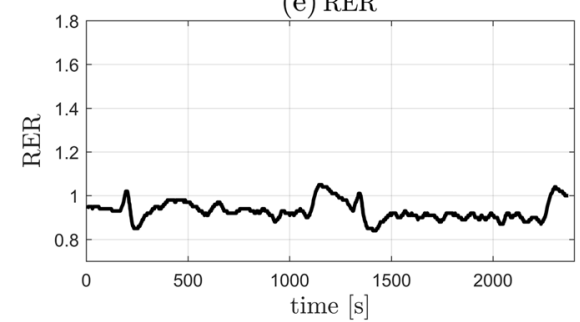

(g) work rate

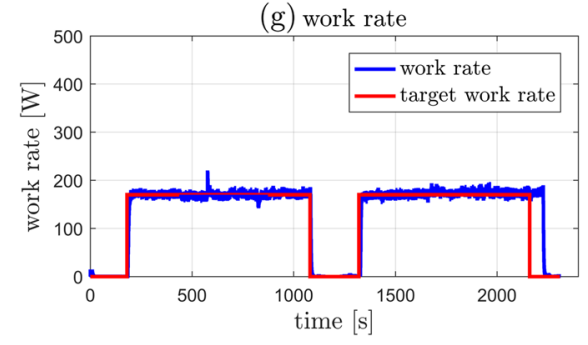

HIIT

(b) oxygen uptake

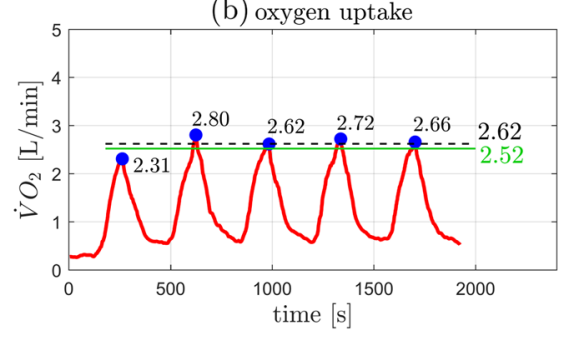

(d) heart rate

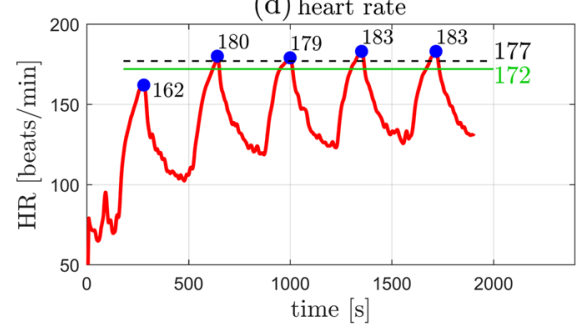

(f) RER

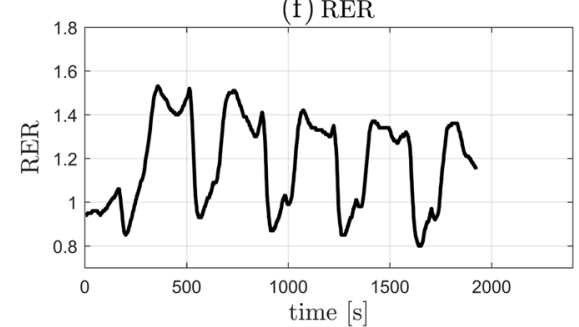

(h) work rate

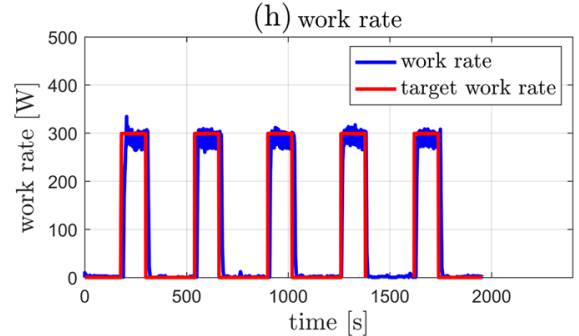

Fig. 5 Original data records from participant 3. Left: CLT, Right: HIIT. (a,b) Oxygen uptake $\dot{V} \mathrm{O}_{2}$. (c,d) Heart rate HR. (e,f) Respiratory exchange ratio (RER). (g,h) Target and measured work rates $\left(P_{\text {target }}, P\right)$. Parts $(\mathbf{a})-(\mathbf{d})$ : the green line indicates the target values, $\dot{V} \mathrm{O}_{2 V T 1}$ for the $\mathrm{CLT}_{\text {and }} \dot{V} \mathrm{O}_{2 V T 2}$ for the HIIT (Table 2); the dashed black line indicates the measured mean value, the steady state mean value for CLT, and the average of the five peak values for HIIT (Table 1). Parts (b),(d): the blue dots indicate the peak values of each interval during HIIT 
Respiratory variables and heart rate were monitored and recorded using a breath-by-breath cardiopulmonary monitoring system (Metamax 3B, Cortex Biophysik $\mathrm{GmbH}$, Germany). Pressure, volume and gas concentration were calibrated prior to each test according the manufacturer's instructions: pressure was calibrated using a certified atmospheric pressure device; volume using a $3 \mathrm{~L}$ syringe; and gas concentrations were calibrated using ambient air and a precision gas mixture $\left(15 \% \mathrm{O}_{2}, 5 \% \mathrm{CO}_{2}\right)$. Heart rate was recorded using a chest belt (T34, Polar Electro Oy, Finland). Analysis of the cardiopulmonary data was done using the proprietary software associated with the breath-by-breath system (Metasoft, version 3.9.9 SR5).

\section{Work rate estimation and targets}

During the training sessions, the participant's feet were fixed on footplates, and his work rate was estimated using force and velocity data (Fig. 2). The work rate estimation algorithm was implemented in Matlab/Simulink (The MathWorks, Inc., USA). The subject's work rate $(P)$ was estimated using force and velocity data provided by the force and angel sensors, and using the following equation:

\section{CLT}

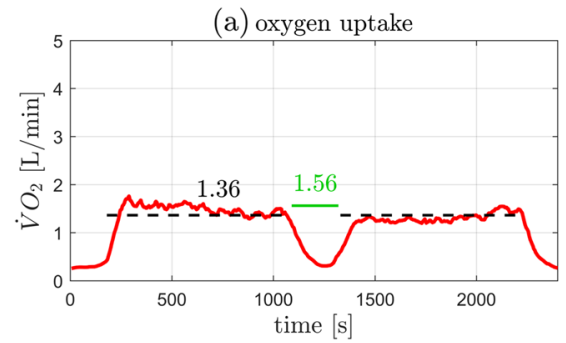

(c) heart rate

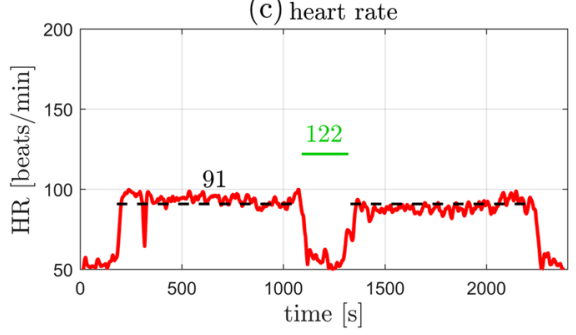

(e) RER

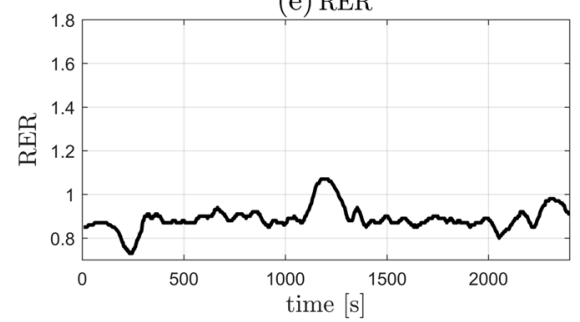

(g) work rate

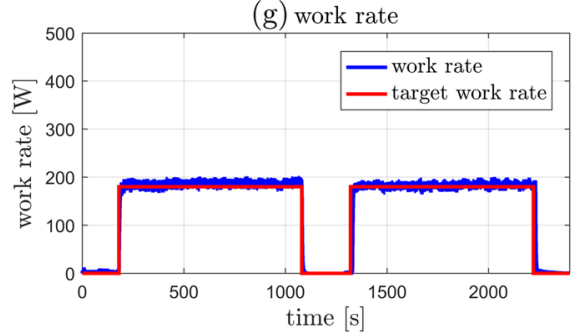

HIIT

(b) oxygen uptake

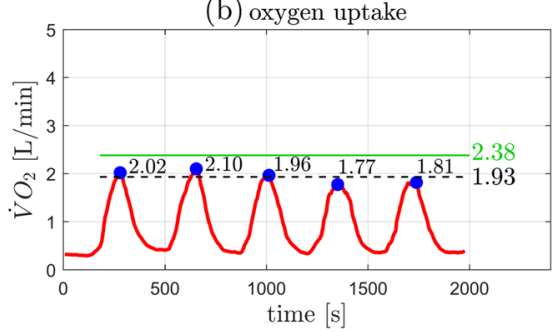

(d) heart rate

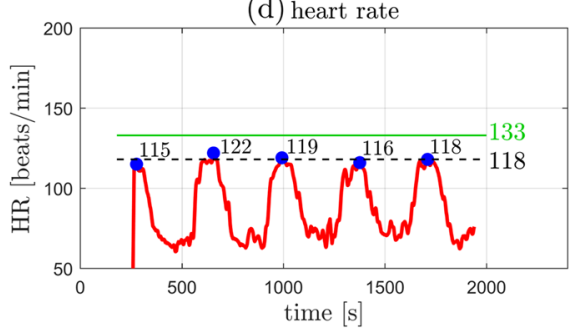

(f) RER

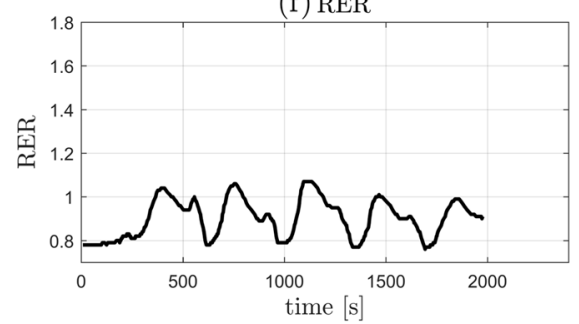

(h) work rate

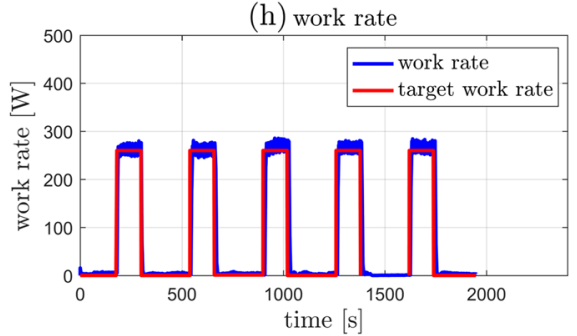

Fig. 6 Original data records from participant 4. Left: CLT, Right: HIIT. (a,b) Oxygen uptake $\dot{V} \mathrm{O}_{2}$. (c,d) Heart rate HR. (e,f) Respiratory exchange ratio (RER). (g,h) Target and measured work rates $\left(P_{\text {target }}, P\right)$. Parts $(\mathbf{a})-(\mathbf{d})$ : the green line indicates the target values, $\dot{V} \mathrm{O}_{2 V T 1}$ for the $\mathrm{CLT}_{\text {and }} \dot{V} \mathrm{O}_{2 V T 2}$ for the HIIT (Table 2); the dashed black line indicates the measured mean value, the steady state mean value for CLT, and the average of the five peak values for HIIT (Table 1). Parts (b),(d): the blue dots indicate the peak values of each interval during HIIT 


$$
\begin{aligned}
P & =P_{1}+P_{\mathrm{r}} \\
& =F_{1} R \dot{\theta}_{1}+F_{\mathrm{r}} R \dot{\theta}_{\mathrm{r}}
\end{aligned}
$$

where $P_{1}$ and $P_{\mathrm{r}}$ are the work rates of the left and right legs, $F_{\mathrm{l}}$ and $F_{\mathrm{r}}$ are the forces applied by the left and right legs, $R$ the radius of the pedal, and $\dot{\theta}_{\mathrm{l}}$ and $\dot{\theta}_{\mathrm{r}}$ are the left and right pedal's angular velocity [18]. Subjects were asked to adjust their volitional effort to maintain the target work rate.

Based on the personal sub-maximal outcomes obtained from the incremental tests performed in the previous study [18], CLT and HIIT protocols were set individually for each participant: for the CLT, the target work rate $P_{\mathrm{VT} 1}$ is the work rate during the ramp phase of the incremental test at the time the first ventilatory threshold occurred. For the HIIT, the target work rate $P_{\mathrm{VT} 2}$ is the work rate at the time of the second ventilatory threshold. Participants were required to adapt their volitional leg effort to keep the estimated work rate as close as possible to the target work rate, and to maintain a constant stepping cadence of $60 \mathrm{steps} / \mathrm{min}$ following the tones of a metronome.

\section{Outcome measures and data analysis}

For the CLT, outcome measures were steady-state levels of $\dot{V} \mathrm{O}_{2}$ and HR: $\dot{V} \mathrm{O}_{2}$ is the mean oxygen uptake obtained during the active phases with a 15 -breath moving average.

\section{CLT}

(a) oxygen uptake

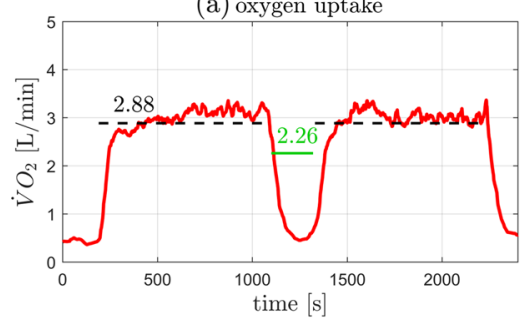

(c) heart rate

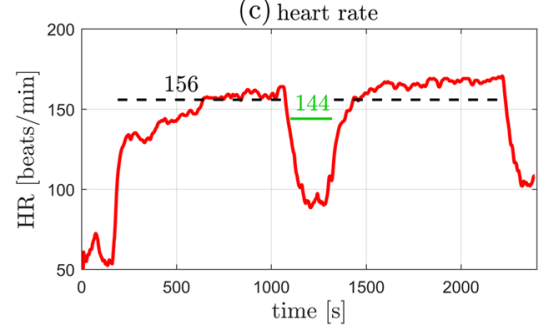

(e) RER

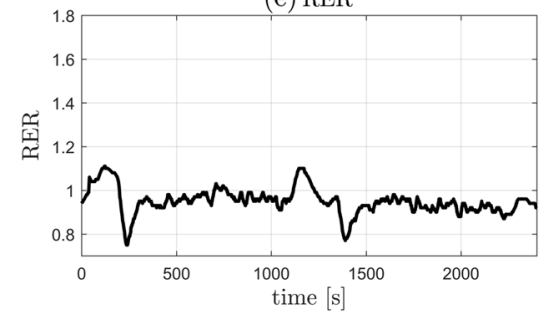

(g) work rate

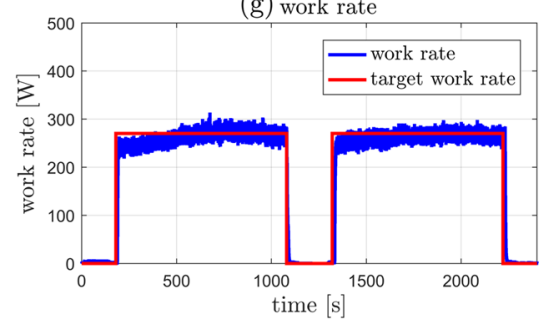

HIIT

(b) oxygen uptake

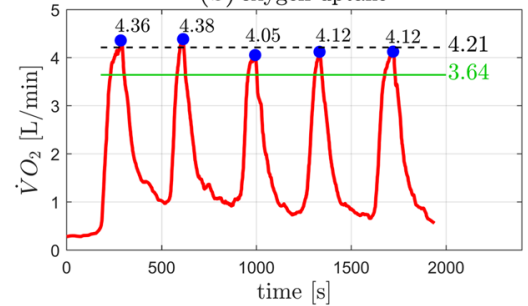

(d) heart rate

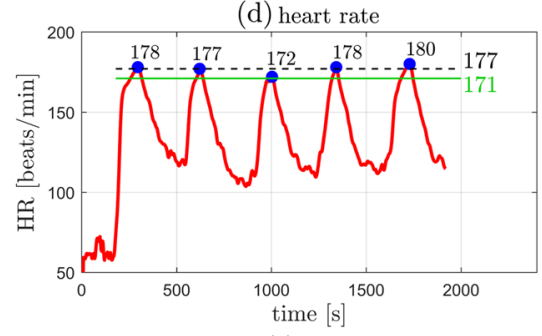

(f) RER

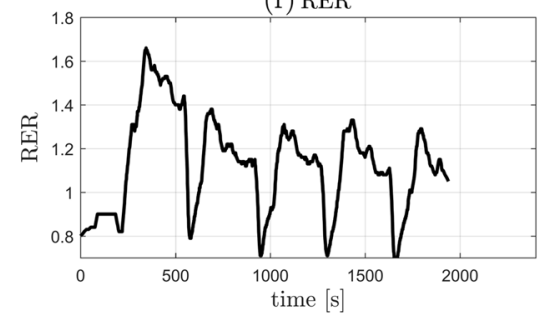

(h) work rate

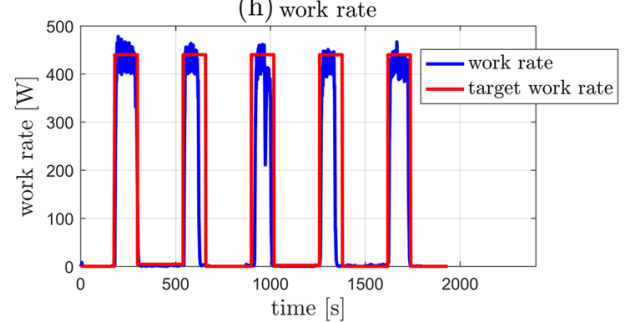

Fig. 7 Original data records from participant 5. Left: CLT, Right: HIIT. (a,b) Oxygen uptake $\dot{V} \mathrm{O}_{2}$. (c,d) Heart rate HR. (e,f) Respiratory exchange ratio (RER). (g,h) Target and measured work rates $\left(P_{\text {target }}, P\right)$. Parts $(\mathbf{a})-(\mathbf{d})$ : the green line indicates the target values, $\dot{V}_{2} \mathrm{O}_{211}$ for the $\mathrm{CLT}_{\text {and }} \dot{V} \mathrm{O}_{2 \mathrm{VT} 2}$ for the HIIT (Table 2); the dashed black line indicates the measured mean value, the steady state mean value for CLT, and the average of the five peak values for HIIT (Table 1). Parts (b),(d): the blue dots indicate the peak values of each interval during HIIT 
$\mathrm{HR}$ is the mean heart rate obtained during the active phases.

For the HIIT, outcome measures were the peak values obtained during the active intervals: $\dot{V} \mathrm{O}_{2}$ is the average of the five peak oxygen uptake values obtained from each interval, using a 15-breath moving average. HR is the average of the five peak heart rate values obtained from each interval. For both protocols, the respiratory exchange ratio RER $=\dot{V} \mathrm{CO}_{2} / \dot{V} \mathrm{O}_{2}$ was recorded with a 15-breath moving average.

Descriptive analyses were performed on all variables. To assess the results obtained from the training sessions of each participant, comparison of training results and incremental test results were performed: for CLT, the mean values of steady-state levels were compared to the corresponding values of $\dot{V} \mathrm{O}_{2 \mathrm{VT} 1}$ obtained from the incremental test; for HIIT, means of the 5 highest values were compared to the corresponding values of $\dot{V} \mathrm{O}_{2 \mathrm{VT} 2}$ obtained from the incremental test. All analyses and displays were performed using MetaSoft and Matlab.

Individual outcome measures were recorded for each participant and are presented in "Results" section (Figs. 3, 4, 5, 6, and 7). For the CLT protocol, the target values $\left(\dot{V} \mathrm{O}_{2 \mathrm{VT} 1}\right.$ and $\left.\mathrm{HR}_{\mathrm{VT} 1}\right)$ are presented with a green line, and the mean steady state value with a dashed black line; for the HIIT protocol, the green line represents the target values $\left(\dot{V} \mathrm{O}_{2 \mathrm{VT} 2}\right.$ and $\left.\mathrm{HR}_{\mathrm{VT} 2}\right)$ and the dashed black line represents the average of the 5 peak values obtained from the intervals (the blue dots).

\section{Feasibility assessment}

The criteria employed for feasibility assessment of cardiopulmonary training using a dynamic leg-press were as follows [29]: (i) implementation - was the approach technically feasible for cardiopulmonary training?; (ii) acceptability - were the training protocols tolerable?; and (iii) responsiveness - was the approach able to provoke substantial cardiopulmonary responses in relation to individual peak values?

\section{Abbreviations}

bpm: beats per minute; $C E$ : cycle ergometer; Cl: confidence interval; DLP. dynamic leg press; HR: heart rate; $H R_{\text {peak: }}$ peak heart rate; MD: mean difference; $P$ : work rate (synonymous with "power"); $\mathrm{P}_{\mathrm{ET}} \mathrm{O}_{2}$ : partial pressure of end-tidal oxygen tension; $\mathrm{PET}_{\mathrm{ET}} \mathrm{CO}_{2}$ : partial pressure of end-tidal carbon dioxide tension; $P_{\text {target: }}$ target work rate; RER: respiratory exchange ratio, $\mathrm{RER} \triangleq \dot{V} \mathrm{CO}_{2} / \dot{V}_{2}$; $R E R_{\text {peak: }}$ peak respiratory exchange ratio; SD: standard deviation; $\dot{V} E$ : minute ventilation; $\dot{V}_{2} \mathrm{VT}_{1}$ : oxygen uptake at the first ventilatory threshold; $\dot{\mathrm{V}} \mathrm{O}_{2 \mathrm{VT} 2}$ : oxygen uptake at the second ventilatory threshold; $\dot{V} \mathrm{O}_{2 \text { peak: }}$ peak oxygen uptake; $\dot{V} \mathrm{O}_{2}$ : rate of oxygen uptake (referred to simply as "oxygen uptake"); $\dot{V} \mathrm{CO}_{2}$ : rate of carbon dioxide output; $t_{\text {ramp }}$ : ramp duration; $\dot{V} \mathrm{E} / \dot{V} \mathrm{O}_{2}$ : ventilatory equivalent of oxygen; $\dot{V} E / \dot{V} \mathrm{CO}_{2}$ : ventilatory equivalent of carbon dioxide; $\mathrm{VT} 1$ : first ventilatory threshold; VT2: second ventilatory threshold

\section{Acknowledgements}

Not applicable.

\section{Authors' contributions}

FC, KH and TN designed the study. FC did the data acquisition. FC and KH contributed to the analysis and interpretation of the data. FC wrote the manuscript; $\mathrm{KH}$ and TN revised it critically for important intellectual content. All authors read and approved the final manuscript.

\section{Funding}

Not applicable.

\section{Availability of data and materials}

The datasets used and/or analysed during the current study are available from the corresponding author on reasonable request.

\section{Ethics approval and consent to participate}

This research was performed in accordance with the Declaration of Helsinki. The study was reviewed and approved by the Ethics Committee of the Swiss Canton of Bern (Ref.: Basec-Nr. 2016-01502). All participants provided written, informed consent.

\section{Consent for publication}

Written consent to publish was obtained from the participant shown in Fig. 2.

\section{Competing interests}

The authors declare that they have no competing interests.

Received: 18 March 2019 Accepted: 30 August 2019

Published online: 03 October 2019

\section{References}

1. Blue MNM, Smith-Ryan AE, Trexler ET, Hirsch KR. The effects of high intensity interval training on muscle size and quality in overweight and obese adults. J Sci Med Sport. 2018;21(2):207-12.

2. Huei Phing C, Abu Saad H, Barakatun Nisak MY, Mohd Nasir MT. Effectiveness of physical activity intervention among government employees with metabolic syndrome. J Exerc Sci Fit. 2017;15(2):55-62.

3. Cicek G, Imamoglu O, Gullu A, Celik O, Ozcan O, Gullu E, et al. The effect of exercises on left ventricular systolic and diastolic heart function in sedentary women: Step-aerobic vs core exercises. J Exerc Sci Fit. 2017;15(2):70-5.

4. Riebe D, Ehrman JK, Ligouri G, Megal M, (eds). ACSM's Guidelines for Exercise Testing and Prescription, 10th ed. Philadelphia: Wolters Kluwer Health; 2018

5. Guiraud T, Nigam A, Gremeaux V, Meyer P, Juneau M, Bosquet L. High-intensity interval training in cardiac rehabilitation. Sports Med. 2012;42(7):587-605.

6. Ito S, Mizoguchi T, Saeki T. Review of high-intensity interval training in cardiac rehabilitation. Int Med. 2016;55(17):2329-36.

7. Weston M, Taylor KL, Batterham AM, Hopkins WG. Effects of low-volume high-intensity interval training (HIT) on fitness in adults : a meta-analysis of controlled and non-controlled trials. Sports Med. 2014;44:1005-17.

8. Astorino TA, Schubert MM. Individual responses to completion of short-term and chronic interval training : a retrospective study. PLOS ONE. 2014;9(5).

9. Ribeiro PAB, Boidin M, Juneau M, Nigam A, Gayda M. High-intensity interval training in patients with coronary heart disease : Prescription models and perspectives. Ann Phys Rehabil Med. 2017;60(1):50-7.

10. Ross LM, Porter RR, Durstine JL. High-intensity interval training (HIIT) for patients with chronic diseases. Journal of Sport and Health Science. 2016;5(2):139-44.

11. Perry CGR, Heigenhauser GJF, Bonen A, Spriet LL. High-intensity aerobic interval training increases fat and carbohydrate metabolic capacities in human skeletal muscle. Appl Physiol Nutr Metab. 2008;33(6):1112-23.

12. Binder RK, Wonisch M, Corra U, Cohen-Solal A, Vanhees L, Saner H, et al. Methodological approach to the first and second lactate threshold in incremental cardiopulmonary exercise testing. Eur J Cardiovasc Prev Rehabil. 2008;15(6):726-34.

13. Mezzani A, Hamm LF, Jones AM, McBride PE, Moholdt T, Stone JA, et al. Aerobic exercise intensity assessment and prescription in cardiac rehabilitation. J Cardiopulm Rehabil Prev. 2013;20(3):442-67.

14. Marzolini S, Oh P, Mcllroy W, Brooks D. The feasibility of cardiopulmonary exercise testing for prescribing exercise to people after stroke. Stroke. 2012;43(4):1075-81.

15. Meyer T, Lucía A, Earnest CP, Kindermann W. A conceptual framework for performance diagnosis and training prescription from submaximal 
gas exchange parameters - Theory and application. Int J Sports Med Suppl. 2005;26(1):38-48.

16. Agostoni P, Bianchi M, Moraschi A, Palermo P, Cattadori G, La Gioia R, et al. Work-rate affects cardiopulmonary exercise test results in heart failure. Eur J Heart Fail. 2005;7(4):498-504.

17. Buchfuhrer MJ, Hansen JE, Robinson TE, Sue DY, Wasserman K, Whipp BJ. Optimizing the exercise protocol for cardiopulmonary assessment. J Appl Physiol. 1983;55(5):1558-64.

18. Chrif F, Nef T, Hunt KJ. Investigation of cardiopulmonary exercise testing using a dynamic leg press and comparison with a cycle ergometer. BMC Sports Sci Med Rehabil. 2018;10:5.

19. Marcus RL, Yoshida Y, Meier W, Peters C, LaStayo PC. An Eccentrically Biased Rehabilitation Program Early after TKA Surgery. Arthritis. 2011;2011: $1-10$.

20. Christensen JC, Foreman KB, LaStayo PC. The positive benefits of negative movement patterns following total knee arthroplasty. Geriatr Orthop Surg Rehabil. 2018;9:1-9.

21. Marcus RL, Smith S, Morrell G, Addison O, Dibble LE, Wahoff-Stice D, et al. Comparison of Combined Aerobic and High-Force Eccentric Resistance Exercise With Aerobic Exercise Only for People With Type 2 Diabetes Mellitus. Phys Ther. 2008:88(11):1345-54.

22. Michaut A, Babault N, Pousson M. Specific effects of eccentric training on muscular fatigability. Int J Sports Med. 2004;25(4):278-83.

23. Wild L, Carl HD, Golditz T, Swoboda B, Hotfiel T. How do leg press exercises comply with limited weight bearing? Phys Ther Sport. 2016;22: $1-5$.

24. Chrif F, Nef T, Lungarella M, Dravid R, Hunt KJ. Control design for a lower-limb paediatric therapy device using linear motor technology. Biomed Signal Process Control. 2017;38:119-27.

25. Pels $A E$, Pollock ML, Dohmeier TE, Lemberger KA, Oehrlein BF. Effects of leg press training on cycling, leg press, and running peak cardiorespiratory measures. Med Sci Sports Exerice. 1987;19(1):66-70

26. Caruso FR, Bonjorno JC, Arena R, Phillips SA, Cabiddu R, Mendes RG, et al. Hemodynamic, autonomic, ventilatory, and metabolic alterations after resistance training in patients with coronary artery disease. Am J Phys Med Rehabil. 2017;96(4):226-35.

27. Karlsen T, Aamot IL, Haykowsky M, Rognmo $\varnothing$. High intensity interval training for maximizing health outcomes. Prog Cardiovasc Dis. 2017;60(1): 67-77.

28. Saengsuwan J, Nef T, Hunt KJ. A method for predicting peak work rate for cycle ergometer and treadmill ramp tests. Clin Physiol Funct Imaging. 2016;37(3):1-5.

29. Bowen DJ, Kreuter M, Spring B, Cofta-Woerpel L, Linnan L, Weiner D, et al. How we design feasibility studies. Am J Prev Med. 2009;36(5):452-7.

\section{Publisher's Note}

Springer Nature remains neutral with regard to jurisdictional claims in published maps and institutional affiliations.

Ready to submit your research? Choose BMC and benefit from:

- fast, convenient online submission

- thorough peer review by experienced researchers in your field

- rapid publication on acceptance

- support for research data, including large and complex data types

- gold Open Access which fosters wider collaboration and increased citations

- maximum visibility for your research: over $100 \mathrm{M}$ website views per year

At BMC, research is always in progress.

Learn more biomedcentral.com/submissions 